\title{
Consolidação em Open Design: uma Abordagem Sociotécnica
}

\author{
Deogenes P. da Silva Junior \\ deogenesj@gmail.com \\ Universidade Federal do Paraná \\ Curitiba, Brasil
}

\begin{abstract}
RESUMO
Open Design é o projeto de design no qual processo e produto são abertos, acessíveis e reusáveis. Open Design tem sua inspiração no Open Source, em que indivíduos consolidam diversas contribuições ao código-fonte de software. Esta Consolidação significa uma ação ou processo intencional de transformar algo em outra coisa mais efetiva, coerente, completa ou elaborada. No Open Design, a complexidade da Consolidação é potencializada, pois suas possibilidades vão além de código-fonte, incluindo outras etapas e objetos de design, como cenários, protótipos, entre outros. Esta dissertação de Mestrado investiga a atividade de Consolidação em processos de design a partir de uma perspectiva sociotécnica e propõe meios para apoiar a Consolidação em uma plataforma de Open Design.
\end{abstract}

\section{PALAVRAS-CHAVE}

Consolidação, Open Design, Perspectiva Sociotécnica

\section{INTRODUÇÃO}

Open Design pode ser entendido como um projeto de design realizado de maneira aberta e compartilhada por indivíduos de potenciais contextos diferentes [2]. Open Design possui inspiração no modelo Open Source [12], no qual as contribuições dos indivíduos são normalmente focadas no código-fonte de software, sendo posteriormente consolidadas em um único código.

No Open Design, a contribuição de um participante pode ocorrer em etapas além da implementação e sobre diferentes objetos de design, como ideias, modelos conceituais, protótipos, entre outros, pois o processo de design é aberto [1]. Deste modo, a complexidade de Consolidação é potencializada, incluindo produções em etapas de entendimento de problema, ideação e reúso, abrangendo diversos objetos do processo de design, envolvendo questões culturais e sociais dos participantes, e exigindo ferramental técnico que viabilize o trabalho.

Consolidação significa " $a$ ação ou processo de fazer algo mais forte ou mais sólido", ou ainda "a ação ou processo de combinar um número de coisas em um todo mais efetivo ou coerente" ${ }^{1}$. Portanto, Consolidação envolve uma ação ou processo intencional de combinar, integrar ou transformar algo em outra coisa mais efetiva, coerente, completa ou elaborada.

\footnotetext{
${ }^{1}$ tradução livre: https://en.oxforddictionaries.com/definition/consolidation

Permission to reproduce or distribute, in whole or in part, material extracted from this work, verbatim, adapted or remixed, as well as the creation or production from the content of such work, is granted without fee for non-commercial use, provided that the original work is properly credited.

IHC 2019 - Workshop de Teses e Dissertações, Outubro 21-25, 2019, Vitória, Brasil. In Anais Estendidos do XVIII Simpósio Brasileiro sobre Fatores Humanos em Sistemas Computacionais. Porto Alegre: SBC.

(C) 2019 by the author(s), in accordance with the terms of the Creative Commons Attribution-NonCommercial 4.0 International Public License (CC BY-NC 4.0).
}

\author{
Roberto Pereira \\ rpereira@inf.ufpr.br \\ Universidade Federal do Paraná \\ Curitiba, Brasil
}

Na literatura, a Consolidação é vista normalmente como um desafio de automação na mesclagem de códigos ou modelos, ficando restrita apenas aos estágios de modelagem e implementação de software. Poucos trabalhos investigam a Consolidação como um desafio do processo de design, composto não somente de aspectos técnicos (e.g., desempenho), mas também de aspectos humanos e organizacionais. Um destes trabalhos investiga a Consolidação de problemas de usabilidade, verificando as decisões tomadas por avaliadores para o "merge" dos problemas identificados [6].

A ótica da Interação Humano-Computador (IHC) nos fornece a visão da Consolidação enquanto um fenômeno humano que pode ser apoiado por ferramentas computacionais [8]. No Open Design, este fenômeno de Consolidação se realiza em um contexto heterogêneo, intermediado por participantes com diversos aspectos formais e informais, com realização de atividades predominantemente colaborativas. Neste contexto, esta pesquisa de Mestrado busca investigar a Consolidação em Open Design de uma perspectiva sociotécnica e propor meios de apoiar esta Consolidação.

\section{PROPOSTA DE TRABALHO}

Entendendo a pesquisa em IHC de uma perspectiva de resolução de problemas, um problema de pesquisa pode ser a falta de compreensão sobre algum fenômeno no uso humano da computação ou a incapacidade declarada de construir uma tecnologia interativa para abordar esse fenômeno para fins desejados [8]. Esta pesquisa lida com a falta de entendimento sobre a Consolidação, tanto de seus pontos importantes de análise quanto da produção de ferramentas computacionais de Consolidação para um projeto de Open Design.

Diferentes visões para Consolidação podem ser encontradas na Literatura. Law e Hvannberg [6] discutem o conceito de Consolidação na área de avaliação de usabilidade e indicam que a prática de Consolidação é aberta e não estruturada. A Consolidação também é diretamente relacionada à ação de merge. Uchitel e Chechik [11] consideram merge como um processo de compor duas descrições parciais de um mesmo componente para obter uma versão mais elaborada das descrições parciais originais. Rubin e Chechik [10], por sua vez, caracterizam um processo de merge em diferentes estágios (compare, match e compose). Outras publicações trazem o conceito de merge ou Consolidação sem definições ou referenciais teóricos sobre o conceito abordado, indicando que não há uma definição dominante ou amplamente adotada na literatura.

Considerando a relevância e os desafios da Consolidação no contexto colaborativo e aberto de Open Design, tem-se como questão principal de pesquisa: "Como apoiar a atividade de Consolidação em Open Design em uma perspectiva sociotécnica?”. Como objetivo geral esta pesquisa busca investigar a Consolidação em Open Design de uma perspectiva sociotécnica. Deste objetivo geral, deriva-se objetivos específicos: 1) entender as características-chave da Consolidação em Open Design; 2) elaborar um Esquema Conceitual para 
entender a Consolidação como um bloco básico de Open Design; 3) investigar e propor meios de apoiar a Consolidação em Open Design na perspectiva sociotécnica; 4) identificar a relação da Consolidação com outros blocos básicos de Open Design.

Bloco básico significa "uma unidade básica de que algo é construído" ${ }^{2}$. Como exemplo tem-se os blocos básicos de software social [9]. Os blocos básicos podem ser compostos de nome, descrição, implicações, exemplos ilustrativos e relações com outros blocos, empregados em uma narrativa para caracterizá-lo, apresentar ressalvas e para promover reflexões para algum propósito.

Nesta pesquisa de mestrado, utiliza-se da base teórica da Semiótica Organizacional (SO) [7] para investigar Consolidação de uma perspectiva sociotécnica e para entendê-la como um bloco básico de Open Design. A SO lida com sistemas interativos de forma a balancear aspectos tecnológicos e sociais, em uma realidade construída socialmente, baseada no comportamento governado por signos dos agentes que dela participam [4]. A SO oferece métodos e artefatos para apoiar o entendimento de problema, modelagem semântica e análise de normas, como a Cebola e Escada Semiótica.

A Cebola Semiótica estrutura uma organização ou grupo social como um sistema de informação composto do nível técnico (design de tecnologia), formal (leis, regras e procedimentos) e informal (crenças, comportamento, valores) do domínio. A Escada Semiótica, por sua vez, representa seis camadas de significado que devem ser consideradas no design de um sistema, sendo: mundo social (consequência de uso de signos nas atividades humanas), pragmática (uso intencional de signos e o comportamento de seus agentes), semântica (relações entre um signo e aquilo a que se refere), sintática (combinação de signos), empírico (propriedades estáticas dos signos) e mundo físico (aspectos físicos dos signos). Por meio destes dois artefatos, pode-se entender o fenômeno de Consolidação como uma atividade sociotécnica, composta de elementos humanos e técnicos.

\section{METODOLOGIA}

Em uma classificação de tipos de pesquisa em IHC [8], a pesquisa Construtiva busca produzir entendimento sobre a construção de um artefato interativo para algum propósito humano no uso da computação. Esta pesquisa pode ser classificada como Construtiva, ao investigar a Consolidação em Open Design de uma perspectiva sociotécnica e propor meios de apoiá-la em uma plataforma técnica. O objetivo não é a construção em si, mas as ideias ou princípios que ela manifesta [8]. Neste caráter construtivo, a pesquisa possui um caráter conceitual na medida em que trabalha com dimensões e conceitos em definição (Consolidação e Open Design).

Nesta classificação Construtiva, a pesquisa assume um caráter exploratório e incremental. A avaliação da pesquisa deve concentrar no entendimento de dimensões do fenômeno de Consolidação a partir de estudos de caso realizados em diferentes contextos, com dados primariamente qualitativos. O entendimento de Consolidação avança enquanto a pesquisa é desenvolvida nos estudos de caso e nas atividades construtivas de design da proposta. Ao final do processo, espera-se avançar com a capacidade de entendimento e de produção de soluções para a atividade humana de Consolidação em uma perspectiva sociotécnica.

\footnotetext{
${ }^{2}$ tradução livre: https://www.lexico.com/en/definition/building_block
}

Para a condução da pesquisa, diferentes tipos de investigações e intervenções serão realizadas, como investigações empíricas, construtivas e conceituais. A investigação conceitual envolve:

1) Mapeamentos Sistemáticos de Literatura (MSL): a partir da verificação dos poucos trabalhos desenvolvidos em Open Design e do campo abrangente de Consolidação, buscou-se realizar Mapeamentos Sistemáticos de Literatura. No MSL em Open Design o objetivo foi conhecer como se apresenta o cenário da pesquisa em Open Design no campo de sistemas computacionais interativos. As bases de pesquisa utilizadas foram ACM Digital Library, IEEE Xplore, Springer e Scopus, selecionando-se publicações no idioma Inglês. Como critério de inclusão, foram considerados artigos que apresentavam pesquisa focada no tema de Open Design. Como critérios de exclusão, foram excluídas pesquisas que não possuíam foco em Open Design de sistemas computacionais interativos, resumos de conferências ou editoriais, artigos fora da língua inglesa, artigos não acessíveis, artigos duplicados e literatura cinzenta (teses, dissertações, monografias). A pesquisa retornou 792 publicações e 12 destas foram selecionadas para o conjunto final de artigos.

No MSL sobre Consolidação, o objetivo foi conhecer e caracterizar a Consolidação no processo de design ou de desenvolvimento de software. Utilizou-se da base de pesquisa $A C M$, com publicações na língua inglesa. Como critério de inclusão, foram considerados artigos que discutiam algum aspecto de Consolidação no processo de design ou de desenvolvimento de software. Como critérios de exclusão, foram excluídos artigos que não tratavam de nenhum aspecto de Consolidação, resumos de conferências, editoriais ou workshops, artigos fora da língua inglesa, artigos não acessíveis, duplicados e literatura cinzenta. A busca retornou 433 publicações, sendo 19 selecionadas para o conjunto final do mapeamento.

2) Análise qualitativa inspirada por Grounded Theory: para identificar dimensões de Open Design e Consolidação, realizou-se a leitura e análise de parágrafos selecionados com aspectos importantes nas publicações do MSL. Inspirado pela técnica de Grounded Theory [5], os aspectos extraídos foram representados em categorias, relacionados e agrupados em termos mais abrangentes, visando encontrar aspectos que diziam sobre a mesma importância, ou que tinham recebido denominações semelhantes (e.g., cooperação e colaboração). Especificamente para a Consolidação, o propósito foi identificar informações que permitissem caracterizar o que era a Consolidação e qual seu significado enquanto um bloco básico de Open Design.

3) Elaboração de um Esquema Conceitual: o referencial metodológico da SO deve ser utilizado para apoiar a reflexão e concepção da Consolidação enquanto um bloco básico. A Cebola Semiótica contribui com a divisão do fenômeno de Consolidação, de modo a identificar quais aspectos podem ser operacionalizados (formal) e quais não são diretamente operacionalizados, mas são aspectos humanos (informal) e influenciam no sistema técnico. A Escada Semiótica pode auxiliar a estruturar o Esquema Conceitual em diferentes camadas de análise, desde os requisitos técnicos de uma plataforma de Open Design (infraestrutura, armazenamento), até as estratégias informais para promover a Consolidação.

A investigação empírica desta pesquisa de Mestrado é composta de estudos de caso exploratórios, que são utilizados para explorar qualquer fenômeno nos dados que serve como um ponto de interesse para o pesquisador [13]. Nesta pesquisa, os estudos de caso foram: 
1) Oficina Participativa: uma oficina participativa de Design Socialmente Consciente [4] foi realizada para produção de soluções para um problema socialmente relevante de fonoaudiologia no contexto hospitalar. Foi realizado o preenchimento colaborativo de artefatos da SO para entendimento do domínio do problema e uma etapa de ideação colaborativa por meio de Brainwriting. Cerca de 10 pessoas participaram da oficina, sendo dois professores e os restantes estudantes de graduação e pós-graduação. Foi verificado que a Consolidação ocorre de maneira incremental, na qual produz-se elementos consolidados que podem ser retomados novamente para Consolidação em outro momento. Como exemplo de uma prática específica de Consolidação na oficina tem-se a Consolidação de ideias ao final de um processo de Brainwriting. Cada participante tinha uma lista de ideias produzidas, na qual deveria ser selecionada as ideias mais relevantes ou que chamaram mais sua atenção. Essas ideias eram comunicadas para o grupo para serem discutidas. Um mediador registrava as ideias que eram apresentadas, agrupando ideias semelhantes ou organizando-as por categorias representativas (funcionalidades, narrativas etc). Para cada ideia apontada poderia ocorrer discussão com prós e contras que decidiriam a inclusão da ideia em uma lista consolidada para a solução.

2) Disciplina de Engenharia de Requisitos (ER): estratégias de Consolidação foram observadas em um processo de Design Thinking em uma disciplina de ER com cerca de 57 estudantes. O projeto prático da disciplina possuía atividades colaborativas para construção de uma solução computacional. As atividades envolviam trabalhos individuais que deveriam ser consolidados colaborativamente, ou a Consolidação a partir do reuso. Foram identificadas estratégias como: um processo sistemático de analisar todos os pontos e discutilos para a composição do elemento consolidado; hipotetização e exploração para oferecer planos de quais consolidações deveriam ser produzidas; apresentar prós e contras, implicando em discussões e em processo decisório; adição, descarte e mescla de produções; e priorização de quais elementos deveriam ser consolidados. Também observamos que a Consolidação pode atuar sob diversos objetos de design como descrições de ideias de um projeto, protótipos gráficos e no reuso de elementos (requisitos, casos de uso etc). A Consolidação ocorreu também em diversas etapas, sejam elas de ideação (Brainwriting) ou mais ligadas à prototipação (Braindrawing).

3) Avaliação com Especialistas: um estudo de caso exploratório será conduzido para a avaliação da proposta do Esquema Conceitual com especialistas de IHC. Estes especialistas estão investigando o conceito de Open Design e propondo uma plataforma técnica para este conceito no "Projeto OpenDesign" [3], do Instituto de Computação - Universidade de Campinas, no qual esta pesquisa de dissertação está situada. Os especialistas podem aplicar o Esquema na plataforma, de modo a derivar requisitos de Consolidação. Por meio da análise do material produzido e de questionários, pode-se verificar a abrangência, utilidade e facilidade de uso do Esquema.

\section{RESULTADOS PRELIMINARES E CENÁRIOS PROSPECTIVOS}

No MSL sobre Consolidação, observa-se que a Consolidação aparece caracterizada por dois momentos principais: o merge e a negociação (processo decisório humano de chegar a um consenso e resolver conflitos). A Consolidação também é caracterizada por aspectos que tratam sobre sua natureza e sobre o entorno de como é realizada (estratégias, negociação, conflitos e ferramentas). Diversas estratégias de Consolidação foram apontadas, como match, merge e diff, entre outras. Entretanto, não foi mencionado na literatura de que maneira todo o processo de Consolidação deve ser realizado, considerando a Consolidação de forma completa: desde o objeto a ser consolidado, passando pelas fases de negociação, de resolução de conflitos, de produção de rationale e de modificação ou merge propriamente dito.

A partir do MSL em Open Design, foi elaborada uma caracterização para Open Design e a derivação de seus blocos básicos, como a Consolidação. Além da Consolidação, alguns exemplos destes blocos são a Comunicação: responsável pelo apoio a diferentes modos de comunicação, com disponibilidade e inclusão; e Compartilhamento: responsável por determinar mecanismos para disponibilização de informações e produtos de design de forma transparente, atendendo à diversidade presente em um ambiente Open Design. Estes blocos se propõem a representar um conjunto abrangente de elementos necessários para viabilizar uma plataforma que apoie a condução de um Open Design.

E a partir do MSL em Consolidação e dos estudos de caso exploratórios, foi derivado um conjunto de dimensões para a caracterização da Consolidação. Enquanto uma atividade, a Consolidação e suas dimensões assumiram uma natureza de operação. Cerca de 7 dimensões principais foram identificadas e articuladas em um Esquema Conceitual preliminar, ilustrado na Figura 1.

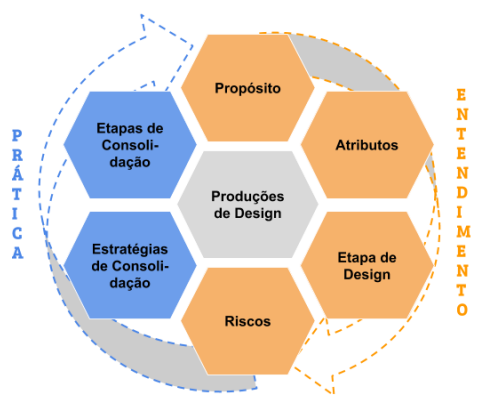

Figura 1: Esquema Conceitual da Consolidação.

No centro da Figura 1 há a dimensão de Produções de Design, que é o elemento manipulado para produzir um objeto final consolidado. As Produções podem ser criadas e assumir diversas naturezas em um processo de design. Nas bordas da figura estão representadas dimensões associadas a uma natureza de entendimento ou prática da Consolidação. As dimensões de entendimento são:

Propósito da Consolidação: o ato de Consolidação normalmente parte de um propósito de consolidar.

Atributos: a Consolidação pode possuir atributos para o "como" deve ser realizada e sobre "o quê" será consolidado.

Etapas de Design: a Consolidação pode ocorrer em determinadas etapas do próprio processo de design. Por exemplo, uma equipe de projeto de design deseja realizar a Consolidação apenas na etapa de Entendimento de Problema.

Riscos: a Consolidação nem sempre pode levar a um resultado mais efetivo, completo ou coerente. Há riscos (e.g., Inconsistência, 
Conflito) que podem levar a atividade de Consolidação para um resultado não desejado. Estes riscos devem ser identificados antes do início da Consolidação para que sejam mitigados.

Além destas quatro dimensões anteriores de entendimento da Consolidação, tem-se as dimensões de prática, sendo:

Estratégias: a Consolidação possui estratégias para organização ou modificação de alguma produção de design. As estratégias de organização são compostas de operações como Match (encontrar similaridades entre produções de design), Diff (encontrar diferenças entre produções de design), Highlight (destacar algum elemento ou atributo de uma produção de design), Rank (ordenar ou priorizar uma lista de produções de design) e Filtering (definir critérios para apresentação de informações). A modificação é constituída de Merge (mesclagem ou unificação de produções), Slice (produzir uma versão alternativa do objeto, baseado em critérios) e Override (processo de sobreposição de alguns elementos em outros no objeto consolidado).

Etapas de Consolidação: a própria Consolidação pode ser vista como constituinte de diversas etapas para sua execução, sendo: a) Organização, b) Seleção, c) Discussão e Negociação, e d) Modificação. Organização se refere a organizar a informação envolvida na Consolidação de modo a colaborar com o entendimento e reduzir o esforço cognitivo envolvido no "fazer sentido" das produções de design. Seleção significa selecionar quais produções de design ou atributos farão parte do objeto final consolidado. A etapa de Discussão e Negociação representa o ajuste e conciliação de várias opiniões de design envolvidas na Consolidação. Por fim, Modificação significa modificar efetivamente as produções de design para refletir um estado de Consolidação planejado.

Estas dimensões podem ser utilizadas para derivar funcionalidades para uma plataforma de Open Design que ofereça e possibilite a Consolidação. Ou podem ser utilizadas para apoiar a reflexão, modelagem e avaliação de soluções de Consolidação, ao favorecer sua divisão em 7 dimensões sobre seu entendimento e prática.

Como contribuições esperadas, tem-se como principal contribuição o Esquema Conceitual a ser produzido que articule as dimensões de Consolidação em Open Design. Também são contribuições as funcionalidades de Consolidação a serem especificadas e implementadas em uma plataforma de Open Design, na medida em que materializam práticas de Consolidação que podem ser aplicadas em outras plataformas.

Em um cenário de prática de design que busca ser mais colaborativo e participativo, a Consolidação torna-se um processo necessário entre os participantes. O Esquema Conceitual de Consolidação tem o potencial de auxiliar projetistas a conceberem um ambiente que facilite que diversos indivíduos mesclem e consolidem suas contribuições, em seu contexto situado de Open Design.

Como limitações, tem-se o MSL de Open Design que considerou apenas publicações no campo de sistemas computacionais interativos. Outras publicações podem ter apresentado elementos importantes, mas estas estavam ligadas às áreas de Engenharia, Desenvolvimento de Produto e Manufatura e, portanto, não foram consideradas. O MSL de Consolidação tem a limitação de incluir apenas uma base de dados $(A C M)$. Considera-se a base foi suficiente para conhecer a Consolidação no contexto computacional, pois $A C M$ é a base mais representativa no contexto de Computação.
O Esquema Conceitual a ser produzido em Consolidação também pode possuir limitações. Enquanto a proposta de Esquema Conceitual busca auxiliar na compreensão e produção de funcionalidades de Consolidação, pode direcionar muito o olhar a determinados pontos, ou deixar outros aspectos de lado. Para redução desta limitação, buscamos realizar estudos de caso em contextos diversos de Consolidação, que aliados aos artefatos da SO, podem oferecer uma visão sistêmica e abrangente para o Esquema Conceitual.

\section{CONCLUSÃO}

Open Design ainda é uma área de pesquisa incipiente no campo de sistemas computacionais interativos. Nos projetos de design que sejam abertos e participativos, a Consolidação se insere como uma atividade relevante na evolução do projeto rumo ao seu propósito definido. A Consolidação também pode ser relevante para o reúso, na medida em que pode facilitar o consolidar de produções de design vindas de outros projetos existentes. Esta pesquisa busca produzir um entendimento sociotécnico de Consolidação enquanto um bloco básico de Open Design. Este entendimento abrange a produção de funcionalidades e a visão da Consolidação enquanto uma atividade humana que pode ser apoiada por ferramentas computacionais. O Esquema Conceitual a ser proposto pode articular dimensões para a realização e sucesso da Consolidação. Um projetista poderia então utilizar este Esquema Conceitual, buscando verificar quais dimensões fazem sentido e como se comportam em seu contexto situado de Open Design.

\section{ACKNOWLEDGMENTS}

Agradecimentos à Coordenação de Aperfeiçoamento de Pessoal de Nível Superior Brasil (CAPES) - Código de Financiamento 001.

\section{REFERÊNCIAS}

[1] Tanja Aitamurto, Dónal Holland, and Sofia Hussain. 2013. Three layers of openness in design: Examining the open paradigm in design research. In DS 75-1: Proceedings of the 19th International Conference on Engineering Design (ICED13). 179-190.

[2] Paul Atkinson. 2011. Orchestral manoeuvres in design. (2011).

[3] M. Cecília C. Baranauskas. 2015. OpenDesign: técnicas e artefatos para o design socialmente consciente de sistemas computacionais. Technical Report. Universidade Estadual de Campinas, Instituto de Computação, Campinas, São Paulo.

[4] Maria Cecília Calani Baranauskas, Maria Cecília Martins, and José Armando Valente. 2013. Codesign de Redes Digitais: tecnologia e educação a serviço da inclusão social. Penso Editora.

[5] Juliet M Corbin and Anselm Strauss. 1990. Grounded theory research: Procedures, canons, and evaluative criteria. Qualitative sociology 13, 1 (1990), 3-21.

[6] Effie Lai-Chong Law and Ebba Thora Hvannberg. 2008. Consolidating usability problems with novice evaluators. In Proceedings of the 5th Nordic conference on Human-computer interaction: building bridges. ACM, 495-498.

[7] Kecheng Liu. 2000. Semiotics in information systems engineering. Cambridge University Press.

[8] Antti Oulasvirta and Kasper Hornbæk. 2016. Hci research as problem-solving. In Proceedings of the 2016 CHI Conference on Human Factors in Computing Systems. ACM, 4956-4967.

[9] Roberto Pereira, M Cecilia C Baranauskas, and SRP Silva. 2010. A discussion on social software: concept, building blocks and challenges. International fournal for Infonomics 3, 4 (2010), 533-542.

[10] Julia Rubin and Marsha Chechik. 2013. N-way model merging. In proceedings of the 2013 9th foint Meeting on Foundations of Software Engineering. ACM, 301-311.

[11] Sebastian Uchitel and Marsha Chechik. 2004. Merging partial behavioural models. In ACM SIGSOFT Software Engineering Notes, Vol. 29. ACM, 43-52.

[12] Ryan Vallance, Sepehr Kiani, and Samir Nayfeh. 2001. Open design of manufacturing equipment. In Proceedings of the CHIRP 1st International Conference on Agile, Reconfigurable Manufacturing. 33-43.

[13] Zaidah Zainal. 2007. Case study as a research method. Furnal Kemanusiaan 5, 1 (2007). 